\title{
Unconventional Superconductivity with a Sign Reversal in the Order Parameter of $\mathrm{LaFeAsO}_{1-x} \mathrm{~F}_{x}$
}

\author{
I. I. Mazin, ${ }^{1}$ D. J. Singh, ${ }^{2}$ M. D. Johannes, ${ }^{1}$ and M. H. Du ${ }^{2}$ \\ ${ }^{1}$ Code 6393, Naval Research Laboratory, Washington, D.C. 20375, USA \\ ${ }^{2}$ Materials Science and Technology Division, Oak Ridge National Laboratory, Oak Ridge, Tennessee 37831-6114, USA
}

(Received 18 March 2008; published 29 July 2008)

\begin{abstract}
We argue that the newly discovered superconductivity in a nearly magnetic, Fe-based layered compound is unconventional and mediated by antiferromagnetic spin fluctuations, though different from the usual superexchange and specific to this compound. This resulting state is an example of extended $s$-wave pairing with a sign reversal of the order parameter between different Fermi surface sheets. The main role of doping in this scenario is to lower the density of states and suppress the pairbreaking ferromagnetic fluctuations.
\end{abstract}

DOI: 10.1103/PhysRevLett.101.057003

PACS numbers: 74.20.- $\mathrm{z}, 74.25 . \mathrm{Jb}, 74.72 .-\mathrm{h}$

The discovery of superconductivity with $T_{c} \geq 26 \mathrm{~K}$ [1] in a compound that contains doped $\mathrm{Fe}^{2+}$ square lattice sheets raises immediate questions about the nature of the superconducting state and the pairing mechanism. A number of highly unusual properties suggest, even at this early stage, that the superconductivity is not conventional. We argue that not only is it unconventional but that doped $\mathrm{LaFeAsO}$ represents the first example of multigap superconductivity with a discontinuous sign change of the order parameter (OP) phase between bands, a state discussed previously (e.g., Refs. [2,3]) but not yet observed in nature.

We suggest that superconductivity here is mediated by spin fluctuations (SFs), as many believe is the case in cuprates, heavy fermion materials, or ruthenates. SFs can induce only a triplet superconducting OP or a singlet one that changes sign over the Fermi surface (FS). The latter condition often, but not always, dictates strong angular anisotropy of the OP (cf. $d$ wave). In our scenario, it is satisfied despite full angular isotropy, since the sign changes between the two sets of FSs. Our model is also principally different from conventional $s$-wave superconductivity discovered in $\mathrm{MgB}_{2}$ : There the pairing interaction is attractive; in our case it is repulsive (but pairing thanks to the sign reversal). Finally, similar to $d$-or $p$-wave superconductivity, our OP has a nearest-neighbor structure in real space, thus reducing the Coulomb repulsion within the pair.

We begin by arguing against conventional superconductivity. The pure compound $\mathrm{LaFeAsO}$ is on the verge of a magnetic instability: It has a very high magnetic susceptibility [1] and is strongly renormalized compared to density functional (DFT) calculations [4]. This renormalization is higher than in any known conventional superconductor, including $\mathrm{MgCNi}_{3}$, where superconductivity is believed to be strongly depressed by spin fluctuations. The susceptibility in the pure compound is large and upon doping with $\mathrm{F}$ grows even larger and becomes Curie-Weiss-like. This suggests nearness to a critical point in the pure compound and nontrivial competition between different SFs. Very strong electron-phonon interactions would be required to overcome the destructive effects of such SFs. We have calculated $a b$ initio the electron-phonon spectral function $\alpha^{2} F(\omega)$ and coupling $\lambda$ for the stoichiometric compound [5]. Some moderate coupling exists, mostly to As modes, but the total $\lambda$ appears to be $\sim 0.2$, with $\omega_{\log } \sim$ $250 \mathrm{~K}\left(\omega_{\log }\right.$ is the logarithmically averaged boson frequency of Eliashberg theory), which can in no way explain $T_{c} \gtrsim 26 \mathrm{~K}$.

The calculated DFT Fermi surfaces [4] for undoped $\mathrm{LaFeAsO}$ consist of two small electron cylinders around the tetragonal $M$ point and two hole cylinders, plus a heavy 3D hole pocket around $\Gamma$. To study doping effects, we performed full-potential calculations using the WIEN package in the virtual crystal approximation and the PerdewBurke-Ernzerhof generalized gradient approximation (GGA) functional [6]. The lattice parameters we took from experiment [1] and optimized the internal positions [7]. In Fig. 1, we show the bands near the Fermi level for $x=0.1$ and the corresponding Fermi surface. As expected, the 3D pocket fills with electron doping (at $x=0.04-0.05$ ) and the fermiology radically simplifies, leaving a highly a)

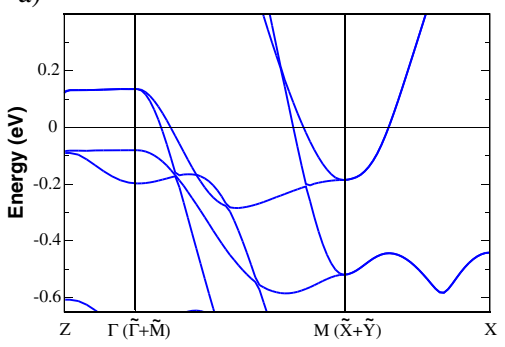

b)

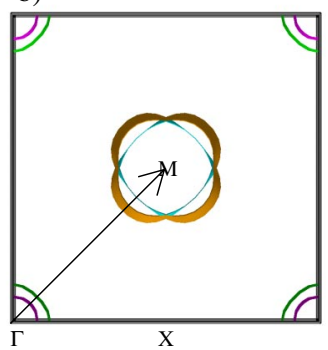

FIG. 1 (color online). (a) Calculated band structure at $x=0.1$ near the Fermi level. (b) Calculated Fermi surface at $10 \%$ doping. Note that the only 3D parts are the far ends of the electron cylinders around $M$. The fully three-dimensional surface present in the undoped compound is suppressed beneath $E_{F}$ by increased electron count. 
2D electronic structure with two heavy hole cylinders and two lighter (and larger) electron cylinders.

This fermiology imposes strong constraints on the superconductivity. In particular, with the exception of phonons, it is hard to identify pairing interactions with a strong $k_{z}$ dependence. Thus, states with strong variations of the OP along $k_{z}$ are unlikely. An angular variation of the OP in the $x y$ plane is possible but would require an unrealistically strong $\mathbf{q}$ dependence of the pairing interaction on the scale of the small Fermi surface size and would also be extremely sensitive to impurities.

On the other hand, the small Fermi surfaces are readily compatible with a pairing state with weak variations of the OP within the sheets but a $\pi$ phase shift between electron and hole cylinders. Here we show that a SF pairing interaction favoring exactly such a state is present in this material, and we discuss the expected consequent physical properties.

The SF spectrum is unusually rich for this compound and comes from three separate sources. First, the system is relatively close to a Stoner ferromagnetic instability. Second, there is a nearest-neighbor antiferromagnetic (AFM) superexchange [8(d)]. Third, there are nestingrelated AFM spin-density-wave-type SFs near wave vectors connecting the electron and hole pockets. The latter appear to be the strongest ones. The corresponding interaction connects the well-separated FS pockets located around $\Gamma$ and around $M$. Though repulsive in the singlet channel, these would nevertheless be strongly pairing provided that the OPs on the two sets of the FSs have opposite signs. The main message of our Letter is that this " $s_{ \pm}$" superconducting state is both consistent with experimental observations and most favored by SFs in this system.

As opposed to the undoped material [4], we do not find any FM solution for the doped compound, even with the more magnetic GGA functional. This suggests that the main function of doping is to move the system away from a ferromagnetic instability (see, however, Ref. [8]). That the system becomes less magnetic is due to the fact that upon doping the heavy 3D hole pocket near $\Gamma$ rapidly fills and the total density of states (DOS) drops (by a factor of 2). At a doping level $x=0.1$, we obtain in the GGA an unrenormalized Pauli susceptibility $\operatorname{Re} \chi_{0}(\mathbf{q}=0, \omega=0) \approx 4 \times 10^{-5} \mathrm{emu} / \mathrm{mole} \quad[N(0)=$ 0.64 states $/ \mathrm{eV} / \mathrm{spin} / \mathrm{Fe}]$. Using $1.1 \mathrm{eV}$ for the Stoner $I$ on $\mathrm{Fe}$, we obtain a renormalized $\chi(0)$ of $0.14 \times$ $10^{-3} \mathrm{emu} / \mathrm{mole}$, a large renormalization but much smaller than what is needed to explain the experimental value [1]. Note that in the undoped system the calculated susceptibility is larger, and the experimental one smaller, than in the doped one. This suggests that besides FM SFs there are other, more important spin excitations in the system.

The first candidate for these is a superexchange corresponding to the standard simple nearest-neighbor checkerboard antiferromagnetism. Importantly, there is also substantial direct Fe-Fe overlap [4], which leads to an additional AFM exchange of comparable strength and with the same checkerboard geometry.

Further discussion requires an understanding of the fermiology in clearer terms. The band structure may at first appear intractably complex, but it is in fact relatively simple, involving only three Fe orbitals near the Fermi level. The hole pockets around $\Gamma$ originate from $\mathrm{Fe} d_{x z}$ and $d_{y z}$ bands that are degenerate at $\Gamma$ and form two nearly perfect concentric cylinders. The electron surfaces are better understood if we recall that the underlying Fe layer forms a square lattice with the period $\tilde{a}=a / \sqrt{2}$ (Fig. 2). The corresponding 2D Brillouin zone (BZ) is twice larger and rotated by $45^{\circ}$. If we could "unfold" the Fermi surface of Fig. 1, we would find the same two hole pockets at $\tilde{\Gamma}$ and one electron pocket around the point $\tilde{X}$ in the large BZ. The latter is formed by the $d_{y z}$ band (or $d_{x z}$ near $\tilde{Y}$ ) that starts $180 \mathrm{meV}$ below the Fermi energy, disperses up along $\tilde{X} \tilde{M}$, and is practically flat along $\tilde{X} \tilde{\Gamma}$. This band is hybridized with the $d_{x y}$ band (or $d_{x^{2}-y^{2}}$ in the two-Fe cell), which starts from $\tilde{X}$ at an energy of $-520 \mathrm{meV}$ and is instead dispersive along $\tilde{X} \tilde{\Gamma}$. Upon hybridization, these two bands yield an oval cylindrical electron pocket, elongated along $\tilde{\Gamma} \tilde{X}[9]$.

Electronic transitions from the hole pockets to the electron pocket should lead to a broad (because the electron pocket is oval rather than circular) peak in the noninteracting susceptibility $\chi_{0}(q, \omega \rightarrow 0)$, at $q=(\pi / \tilde{a}, 0)$, while the superexchange interaction $J(q)$ on the square lattice should be peaked at $q=(\pi / \tilde{a}, \pi / \tilde{a})$ ( $\Gamma$ in the downfolded BZ). The renormalized susceptibility $\chi(q)=\chi_{0}(q) /[1-$ $\left.J(q) \chi_{0}(q)\right]$ then has a rich structure with maxima at $\tilde{\Gamma}, \tilde{X}$, and $\tilde{M}$. For the true unit cell with two Fe, both $\tilde{\Gamma}$ and $\tilde{M}$ fold
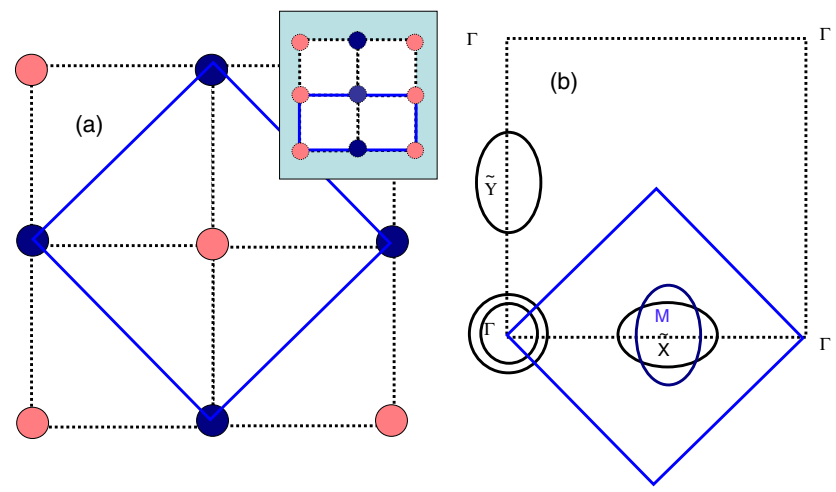

FIG. 2 (color online). Fermi surface formation upon backfolding of the large $\mathrm{BZ}$ corresponding to a simple $\mathrm{Fe}$ square lattice. (a) Real space: the four small unit cells of the Fe-only sublattice (dashed lines) with the larger solid diamond of actual two-Fe unit cell. Dark and light circles indicate superexchange (checkerboard) ordering. The inset shows the spin-density wave corresponding to $\tilde{X}$ point SFs. (b) Reciprocal space: the dashed black square is the unfolded BZ, the solid blue diamond is the downfolded zone, and the blue ellipse is the electron pocket from the $\tilde{Y}$ point downfolded onto the $\tilde{X}$ point (which is $M$ in the small $\mathrm{BZ})$. 
down into the $\Gamma$ point, while $\tilde{X}$ folds down into the $M$ point. The corresponding folding of the Fermi surfaces makes the electron pockets overlap, forming two intersecting surfaces (Fig. 1). It is important to appreciate from this gedanken unfolding that already on the level of the noninteracting susceptibility there is a tendency for antiferromagnetic correlations with a wave vector different from the superexchange one.

In Fig. 3, we present the calculated [10] $\chi_{0}(q, \omega)=$ $\frac{f\left(\epsilon_{k}\right)-f\left(\epsilon_{k+q}\right)}{\epsilon_{k}-\epsilon_{k+q}-\omega-i \delta}$ at $\omega \rightarrow 0$ and $q_{z}=\pi / c$ ( $\chi$ is practically independent of $q_{z}$ ), The peak at $M$, derived from interband transitions, is very broad, as expected, with some structure around the $M$ point, due to the particular orientation of the two oval pockets at $M$ and the size difference between the hole and electron cylinders for finite doping. With minor modification, this structure is present also in the undoped compound.

We have also performed magnetic calculations (discussed in a separate publication) in a supercell corresponding to the superexchange $\mathbf{q}=\tilde{M}(=\Gamma$ in the downfolded Brillouin zone) and nesting-induced $\mathbf{q}=\tilde{X}(=M)$ spindensity waves. We find, indeed, that the tendency to ferromagnetic ordering is suppressed in the doped compound, while the tendency to nesting-based antiferromagnetism is present, even leading to an actual instability at the mean field level. It is worth noting that, while this instability does not appear in actual superconducting materials, it has been now found experimentally in the low doping regime [8].

The strong AFM SFs around $M$ favor our proposed $s_{ \pm}$ state. Cases where SF-induced interactions connect two pockets of the Fermi surfaces, including SFs originating from electronic transitions between the very same pockets have been considered in the past [11]. However, they involved FS pockets related by symmetry, which strongly restricts the phase relations between them. In our case, the two sets of pockets are not symmetry related, and nothing prevents them from assuming arbitrary phases.

For the singlet case, the coupling matrix between the hole $(h)$ and the electron pockets $(e)$ is negative: $\lambda_{\text {eh }}<0$. The diagonal components emerge from competition be-
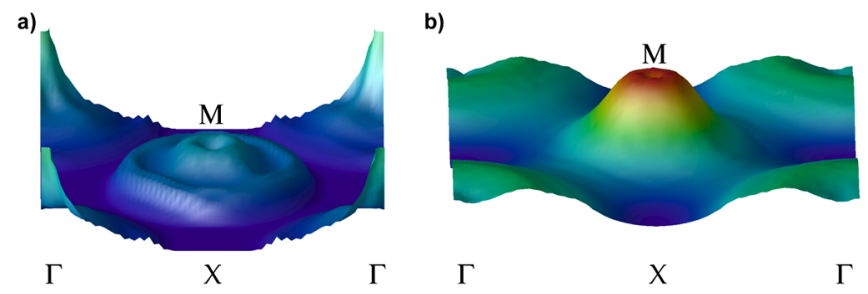

FIG. 3 (color online). The imaginary (a) and the real (b) parts of the noninteracting susceptibility $\chi_{0}(q, \omega \rightarrow 0)$, in arbitrary units. Within common approximations $\operatorname{Im} \chi=\operatorname{Im}\left[\frac{\chi_{0}}{\left(1-J(\mathbf{q}) \chi_{0}(q, w)\right.}\right]$ is measurable by neutron scattering and $\operatorname{Re} \chi$ controls the pairing interaction, in the singlet channel proportional to $1 /[1-$ $J(\mathbf{q}) \chi_{0}(q, 0)$ ] (see Ref. [18] for a review). Note that the RPA enhancement will strengthen both peaks. tween the attractive phonon-mediated and repulsive SFmediated interactions and are, presumably, weak. If $\lambda_{h h}^{\mathrm{ph}}$ is the average of the phonon-mediated interaction over the wave vectors $q<0<2 k_{F}^{h}$, and $\lambda_{h h}^{\mathrm{sf}}$ the same for the SF, then $\lambda_{h h}=\lambda_{h h}^{\mathrm{ph}}-\lambda_{h h}^{\mathrm{sf}}$. Our calculated electron-phonon coupling comes mostly from small wave vectors, that is, $\lambda_{h h}^{\mathrm{ph}}+\lambda_{e e}^{\mathrm{ph}} \approx 0.2$ and $\lambda_{e h}^{\mathrm{ph}} \approx 0$. Phonons, therefore, though weak, promote the $s_{ \pm}$state. On the other hand, $\lambda_{h h}^{\mathrm{sf}}$ and $\lambda_{e e}^{\mathrm{sf}}$ come from FM (small- $q$ ) SFs and are pair-breaking. Finally, the superexchange interaction does not affect $\lambda_{h h}$, but the corresponding wave vector $\mathbf{q}=\tilde{\Gamma} \tilde{M}$ connects the $e$ pockets near $\tilde{X}$ and $\tilde{Y}$, and such fluctuations are also pair-breaking [8(c)].

The $T_{c}$, as usual for a two-gap superconductor [12], is defined by the maximal eigenvalue of the $\lambda$ matrix $\lambda_{\text {eff }}=$ $\left[\lambda_{h h}+\lambda_{e e}+\sqrt{4 \lambda_{e h} \lambda_{h e}+\left(\lambda_{e e}-\lambda_{h h}\right)^{2}}\right] / 2$, and the OPs $\Delta_{e, h}$ are defined by the corresponding eigenvector. In our case, the signs of $\Delta_{h}$ and $\Delta_{e}$ will be opposite, and their absolute values will be similar since presumably $\left(\lambda_{e e}-\right.$ $\left.\lambda_{h h}\right)^{2} \ll \lambda_{e h} \lambda_{h e}$. This " $s_{ \pm}$" state is analogous to the states proposed previously for semimetals [13] and bilayer cuprates [14].

From the point of view of neutron scattering, the structure of the peak in $\chi$ near $M$ is important, but, for SFinduced superconductivity, it does not matter at all. A welldefined spin excitation requires a sharp peak, but the pairing interaction is integrated over all possible $q$ vectors spanning the two sets of Fermi surfaces so that only the total weight of the peak is important. Dimensionality is, however, very important, as 3D coupling supports a longrange magnetic ordering (competing with superconductivity). Indeed, experiment shows an abrupt appearance of superconductivity at $x \approx 0.03$, roughly where the $3 \mathrm{D}$ pockets around $\Gamma$ disappear.

One might envision a triplet state similar to the described singlet one, fully gapped, as expected for unitary 2D $p$-wave states, and with different amplitudes (or signs) on the two cylinders. The similarity, however, is misleading. In the triplet channel, SFs induce attraction, but given the relatively large width of the AF peak (Fig. 3), a large part of the pairing will be lost as only SF with a wave vector exactly equal to $(\pi / a, \pi / a)$ will be fully pairing, and some others will even be pair-breaking. Therefore, in this scenario where the antiferromagnetic SFs around the $M$ point provide the primary pairing interaction, we expect the lowest energy superconducting state to be $s_{ \pm}$. The structure of the OP in real space can be evaluated using the lowest Fourier component, compatible with the proposed $s_{ \pm}$state, namely, $\cos k_{x}+\cos k_{y}$ (equivalently, $\cos \tilde{k}_{x} \cos \tilde{k}_{y}$ ). This corresponds to pairing of electrons that reside on the nearest neighbors, just as in the $d$-wave case, so that the on site Coulomb repulsion is not particularly destructive for this state.

Finally, we discuss the experimental ramifications of the $s_{ \pm}$state. In many aspects they are similar to that of the $s_{ \pm}$ 
state proposed in Ref. [14] and discussed in some detail in Refs. [14-16]. The thermodynamic and tunneling characteristics are the same as for a conventional two-gap superconductor [12]. The two-gap character, however, may be difficult to resolve, given the dominance of the interband interactions which will render the two gap magnitudes similar. Nonmagnetic small- $q$ intraband $(e-e$ or $h-h)$ scattering as well as the interband spin-flip pairing will not be pair-breaking, but paramagnetic interband scattering will, resulting in a finite DOS below the gap [2], consistent with specific heat measurements [17]. The most interesting feature of the $s_{ \pm}$state [16] is that the coherence factors for exciting Bogliubov quasiparticles on FS sheets with opposite signs of the OP are reversed compared to conventional coherence factors. We outline a few important consequences of the relatively straightforward application of this concept to experimental probes. First, one expects a qualitative difference between experiments that probe vertical transitions $(\mathbf{q}=0)$ and those that probe transitions with $\mathbf{q}$ close to $\pi / a, \pi / a$. For instance, the spin susceptibility at $\mathbf{q}=0$ will behave conventionally, i.e., exponentially decay below $T_{c}$ without any coherence peak, while the susceptibility for $\mathbf{q} \approx \pi / a, \pi / a$ will have a coherence peak that should be detectable by neutron scattering as an enhancement below $T_{c}$ [16]. AFM SFs near $\pi / a, \pi / a$ dominate in the doped material, and so the usual coherence peak in the NMR relaxation rate, which averages equally over all wave vectors, is expected to disappear or be strongly reduced. It was shown in Ref. [15] that Josephson currents from FSs with different signs of the OP interfere destructively, and the net phase corresponds to the sign of the FS with the higher normal conductance. In the constant relaxation time approximation, both in-plane and out-of-plane conductivities are dominated by the electron pockets. This is unfortunate, since there would otherwise be a $\pi$ phase shift between the $a b$ and $c$ tunneling, and corner-junction experiments could be used, as in high- $T_{c}$ cuprates.

In summary, we argue that the fermiology found in doped $\mathrm{LaAsFeO}$ gives rise to strong but broad antiferromagnetic spin fluctuations near the $M$ point in the Brillouin zone, while the tendency to magnetism existing at zero doping is suppressed. These fluctuations, while too broad to induce a magnetic instability, are instrumental in creating a superconducting state with OPs of opposite signs on the electron and hole pockets.

We acknowledge helpful discussions with D. Scalapino. Work at NRL is supported by ONR. Work at ORNL was supported by DOE, Division of Materials Science and Engineering.

[1] Y. Kamihara et al., J. Am. Chem. Soc. 130, 3296 (2008)..
[2] A. A. Golubov and I. I. Mazin, Phys. Rev. B 55, 15146 (1997).

[3] D. F. Agterberg, V. Barzykin, and L. P. Gor'kov, Phys. Rev. B 60, 14868 (1999).

[4] D. J. Singh and M. H. Du, Phys. Rev. Lett. 100, 237003 (2008).

[5] We used linear response theory with ultrasoft pseudopotentials as implemented within the QUANTUM ESPRESSO package by S. Baroni et al., http://www.pwscf.org/. We used a zone sampling of 1377 inequivalent $\mathbf{k}$ points, a $4 \times$ $4 \times 2$ phonon mesh, and a plane wave cutoff of $50 \mathrm{Ry}$.

[6] J. P. Perdew, S. Burke, and M. Ernzerhof, Phys. Rev. Lett. 77, 3865 (1996).

[7] The optimized positions actually depend slightly on doping. For $x=0.1$, in GGA, they are $z_{\mathrm{La}}=0.148$ and $z_{\mathrm{As}}=$ 0.638 .

[8] After this manuscript was submitted for publication, several dozen relevant preprints have been posted, in particular, (a) experimental verification of the spin-density wave (SDW) state predicted here (inset in Fig. 2), at $x \leqq 0.05$ (M. A. McGuire et al., arXiv:0804.0796); (b) while we calculated the SDW stripe ordered phase at $x=0.1$, Dong Sun et al., arXiv:0803.2883 have subsequently shown that, contrary to our initial conjecture, at zero doping this instability is even stronger; (c) K. Kuroki et al., arXiv:0803.3325 have studied the SF-induced superconductivity in $\mathrm{LaFeAsO}$ adding the RPA renormalization to our model and found that the $s_{ \pm}$state has the lowest energy as long as the hole pockets are present (otherwise, a nodeless $d$-wave state is the most stable one); and (d) T. Yildirim, arXiv:0804.2252 has pointed out that the nextnearest-neighbor superexchange in this system is of the same order as the nearest-neighbor superexchange, adding a fourth nontrivial magnetic interaction to those we considered. These and other recent results support our main proposition that the role of doping is to drive the system away from a magnetic instability, though we misidentified the offending instability as ferromagnetic.

[9] We thank P. A. Lee for pointing out an erroneous transmutation of $\tilde{X}$ and $\tilde{Y}$ in our original description.

[10] We used a three-dimensional grid of $\approx 75000 k$ and $q$ points with a temperature smearing of $1 \mathrm{mRy}$ and the constant matrix element approximation.

[11] M. D. Johannes, I. I. Mazin, D. J. Singh, and D. A. Papaconstantopoulos, Phys. Rev. Lett. 93, 097005 (2004); K. Voelker and M. Sigrist, arXiv:cond-mat/0208367.

[12] I. I. Mazin and V.P. Antropov, Physica (Amsterdam) 385C, 49 (2003).

[13] A. G. Aronov and E. B. Sonin, Zh. Eksp. Teor. Fiz. 63, 1059 (1972) [Sov. Phys. JETP 36, 556 (1973)].

[14] A. I. Liechtenstein, I. I. Mazin, and O. K. Andersen, Phys. Rev. Lett. 74, 2303 (1995).

[15] I. I. Mazin, A. A. Golubov, and A. D. Zaikin, Phys. Rev. Lett. 75, 2574 (1995).

[16] I. I. Mazin and V. Yakovenko, Phys. Rev. Lett. 75, 4134 (1995).

[17] G. Mu, X. Zhu, L. Fang, L. Shan, C. Ren, and H. H. Wen, arXiv:0803.0928.

[18] T. Moria and K. Ueda, Rep. Prog. Phys. 66, 1299 (2003). 